\title{
openheart Left ventricular longitudinal systolic function analysed by 2D speckle- tracking echocardiography in heart failure with preserved ejection fraction: a meta-analysis
}

Daniel Armando Morris, ${ }^{1}$ Xin-Xin Ma, ${ }^{2}$ Evgeny Belyavskiy, ${ }^{1}$

Radhakrishnan Aravind Kumar, ${ }^{1}$ Martin Kropf, ${ }^{1}$ Robin Kraft, ${ }^{1}$ Athanasios Frydas, ${ }^{1}$ Engin Osmanoglou, ${ }^{3}$ Esteban Marquez, ${ }^{4}$ Erwan Donal, ${ }^{5}$ Frank Edelmann, ${ }^{1}$ Carsten Tschöpe, ${ }^{1}$ Burkert Pieske, ${ }^{1,6}$ Elisabeth Pieske-Kraigher ${ }^{1}$

To cite: Morris DA, Ma X-X, Belyavskiy E, et al. Left ventricular longitudinal systolic function analysed by $2 \mathrm{D}$ speckle-tracking echocardiography in heart failure with preserved ejection fraction: a metaanalysis. Open Heart 2017;4:e000630. doi:10.1136/ openhrt-2017-000630

Dr Morris and Dr Ma contributed equally to this study.

Received 15 March 2017 Revised 23 June 2017 Accepted 18 July 2017

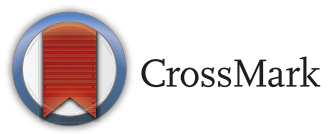

For numbered affiliations see end of article.

\section{Correspondence to} Dr Daniel Armando Morris, Department of Internal Medicine and Cardiology, Charité - Universitätsmedizin Berlin, Berlin, Germany; danielarmando.morris@charite.de

\section{ABSTRACT}

Background The purpose of this meta-analysis was to confirm if the global longitudinal systolic function of the left ventricle (LV) is altered in patients with heart failure with preserved ejection fraction (HFpEF).

Methods We searched in different databases (Medline, Embase and Cochrane) studies that analysed LV global longitudinal systolic strain (GLS) in patients with HFpEF and in controls (such as healthy subjects or asymptomatic patients with arterial hypertension, diabetes mellitus or coronary artery disease).

Results Twenty-two studies (2284 patients with HFpEF and 2302 controls) were included in the final analysis. Patients with HFpEF had significantly lower GLS than healthy subjects (mean $-15.7 \%$ (range $-12 \%$ to $-18.9 \%$ ) vs mean $-19.9 \%$ (range $-17.1 \%$ to $-21.5 \%$ ), weighted mean difference $-4.2 \%(95 \% \mathrm{Cl}-3.3 \%$ to $-5.0 \%)$, p $<0.001$, respectively). In addition, patients with HFpEF had also significantly lower GLS than asymptomatic patients (mean $-15.5 \%$ (range $-13.4 \%$ to $-18.4 \%$ ) vs mean $-18.3 \%$ (range $-15.1 \%$ to $-20.4 \%$ ), weighted mean difference $-2.8 \%(95 \% \mathrm{Cl}-1.9 \%$ to $-3.6 \%), \mathrm{p}<$ 0.001 , respectively). In line, 10 studies showed that the rate of abnormal GLS was significantly higher in patients with HFpEF (mean 65.4\% (range 37\%-95\%)) than in asymptomatic subjects (mean 13\% (range 0\%-29.6\%)). Regarding the prognostic relevance of abnormal GLS in $\mathrm{HFpEF}$, two multicentre studies with large sample size (447 and 348) and high number of events (115 and 177) showed that patients with abnormal GLS had worse cardiovascular (CV) outcomes than those with normal GLS (HR for CV mortality and HF hospitalisation 2.14 (95\% Cl 1.26 to 3.66 ) and 1.94 (95\% Cl 1.22 to 3.07$)$ ), even adjusting these analyses for multiples clinical and echocardiographic variables.

Conclusion The present meta-analysis analysing 2284 patients with HFpEF and 2302 controls confirms that the longitudinal systolic function of the LV is significantly altered in high proportion of patients with HFpEF. Further large multicentre studies with the aim to confirm the prognostic role of abnormal GLS in HFpEF are warranted.

\section{INTRODUCTION}

Heart failure with preserved ejection fraction (HFpEF) has long been considered a disorder characterised principally by left ventricular (LV) diastolic alterations. ${ }^{1-3}$ While it is correct, recent studies using two-dimensional speckle-tracking echocardiography (2DSTE) have suggested that the longitudinal systolic function of the LV is altered in HFpEF. ${ }^{4-26}$ Nonetheless, despite these interesting pathophysiological insights, other studies including old control patients and well-characterised patients with $\mathrm{HFpEF}$ did not find any difference in LV global longitudinal systolic strain (GLS) between HFpEF and controls as well as any clinical relevance of GLS in HFpEF. ${ }^{27-33}$ Accordingly, given these contradictory results, at this time it is difficult to confirm the magnitude of an altered LV longitudinal systolic function in patients with HFpEF. In addition, it remains uncertain the exact rate of abnormal GLS in HFpEF or whether the prevalence of this LV systolic alteration is significantly different to asymptomatic controls. In line, a global examination or meta-analysis addressing all these important issues in HFpEF is lacking.

Therefore, the purpose of this meta-analysis was to analyse the global longitudinal systolic function of the LV in all published studies that included HFpEF and control patients with the aim to confirm if the global longitudinal systolic function of the $\mathrm{LV}$ is altered in patients with HFpEF.

\section{METHODS}

\section{Search process}

We searched in different databases (Medline, Embase and Cochrane) published studies 


\section{KEY QUESTIONS}

What is already known about this subject?

- Heart failure with preserved ejection fraction (HFpEF) has long been considered a disorder characterised principally by left ventricular (LV) diastolic alterations. While it is correct, recent studies using two-dimensional speckle-tracking echocardiography have suggested that the longitudinal systolic function of the $\mathrm{LV}$ is altered in HFpEF. Nonetheless, despite these interesting pathophysiological insights, other studies including old control patients and well-characterised patients with HFpEF did not find any significant difference in LV global longitudinal systolic strain (GLS) between HFpEF and controls. Accordingly, given these contradictory results, at this time it is difficult to confirm the magnitude of an altered LV Iongitudinal systolic function in patients with HFpEF. In addition, it remains uncertain the exact rate of abnormal GLS in HFpEF or whether the prevalence of this LV systolic alteration is significantly different to asymptomatic controls. In line, a global examination or meta-analysis addressing all these important issues in HFpEF is lacking.

What does this study add?

- On the basis of 22 studies, 2284 patients with HFpEF and 2302 controls, the findings of this meta-analysis confirm that patients with HFpEF have significantly lower LV Iongitudinal systolic function than asymptomatic controls and that a longitudinal systolic dysfunction of the LV is common among patients with HFpEF.

How might this impact on clinical practice?

- Several clinical trials have been conducted to restore the diastolic function of the LV in patients with HFpEF with the aim to improve the prognosis of these patients. However, none of these treatments has been shown to decrease mortality in patients with HFpEF. For this reason, additional pathophysiological mechanisms should be taken into consideration in the design of new clinical trials in this heterogeneous disease. The present meta-analysis analysing 2284 patients with HFpEF and 2302 controls confirms that the longitudinal systolic function of the LV is significantly altered in high proportion of patients with HFpEF. In addition, two large multicentre studies showed that an abnormal LV Iongitudinal systolic function is significantly linked to cardiovascular mortality and HF hospitalisation in these patients. Therefore, we consider that further large multicentre studies with the aim to validate the prognostic relevance of an abnormal GLS in patients with HFpEF are warranted, because if the prognostic role of this LV systolic alteration is confirmed, a future therapeutic target could arise on this complex disease, for which, so far, no effective therapies exist.

until 15 June 2017 that analysed the global longitudinal systolic function of the LV using 2DSTE in patients with HFpEF. We searched the following Medical Subject Heading terms: 'heart failure', 'echocardiography' and 'strain'. In addition, we reviewed the citations in the selected articles to search for additional studies.

\section{Selection criteria}

The criteria to include the studies were: (1) patients with diagnosis of HFpEF using a cut-off of left ventricular ejection fraction (LVEF) $\geq 45 \%$; (2) available LV GLS analysed by 2DSTE at rest in at least $12 \mathrm{LV}$ segments and
(3) available control group or data regarding the prevalence of abnormal GLS or data regarding the prognosis of GLS. Control group in the analysis was defined as healthy subjects or as asymptomatic patients with some cardiovascular (CV) risk factor or disease such as arterial hypertension, diabetes mellitus or history of coronary artery disease (CAD). Furthermore, in order to avoid analysing twice the same population, we selected only one study when the same population was included in two or more HFpEF studies for the same research group.

\section{Data abstraction and variable definition}

Data were independently extracted by two reviewers (DAM and X-XM). Clinical characteristics, design, imaging modalities for quantification of GLS, baseline values of GLS in HFpEF and controls, rate of abnormal GLS and hazard ratio (HR) or odds ratio (OR) that linked GLS to CV outcomes were extracted from each study. The key variable under study was GLS (ie, peak systolic LV strain) derived from the myocardial analysis of the $\mathrm{LV}$ in longitudinal direction in the apical 4-chamber, 2-chamber and 3-chamber views (ie, $\geq 12 \mathrm{LV}$ segments) and using 2DSTE at rest.

\section{Statistical analysis}

We used Review Manager (V.5.3, Cochrane) to analyse the data. All analyses were in accordance with the PRISMA-IPD Statement recommendations. ${ }^{34}$ Mean, 95\% confidence interval (CI) and range were calculated for each variable from all studies. In line, we determined the weighted mean difference (WMD) for each variable in each study. A fixed model was used to obtain WMD. Statistical heterogeneity in GLS values among studies was evaluated using the $\mathrm{I}^{2}$ statistics. In addition, we performed a meta-regression analysis in order to detect the possible sources of statistical heterogeneity on GLS values in the study population. Moreover, a sensitivity analysis was performed in order to decrease the possible bias or sources of statistical heterogeneity on GLS. In this regard, we performed subgroup analyses including studies with $\geq$ 100 patients with $\mathrm{HFpEF}$ and studies with $<100$ patients with HFpEF as well as studies with patients with HFpEF without atrial fibrillation. Furthermore, with the purpose of evaluating the association of GLS with CV outcomes in HFpEF, we analysed the link of GLS to CV outcomes analysing the OR and HR in logistic and Cox regression analysis in the studies. Differences were considered statistically significant when $\mathrm{p}$ value was $<0.05$.

\section{RESULTS}

\section{Study population}

We identified 953 potential studies from published literature (see figure 1). Twenty-nine studies met the eligibility criteria analysing the different databases (Medline, Embase and Cochrane) (see table 1). Twenty-two studies had a control group (2284 patients with HFpEF and 2302 controls) and nine studies had follow-up with outcomes analyses (1847patients with HFpEF) (see table 1). 


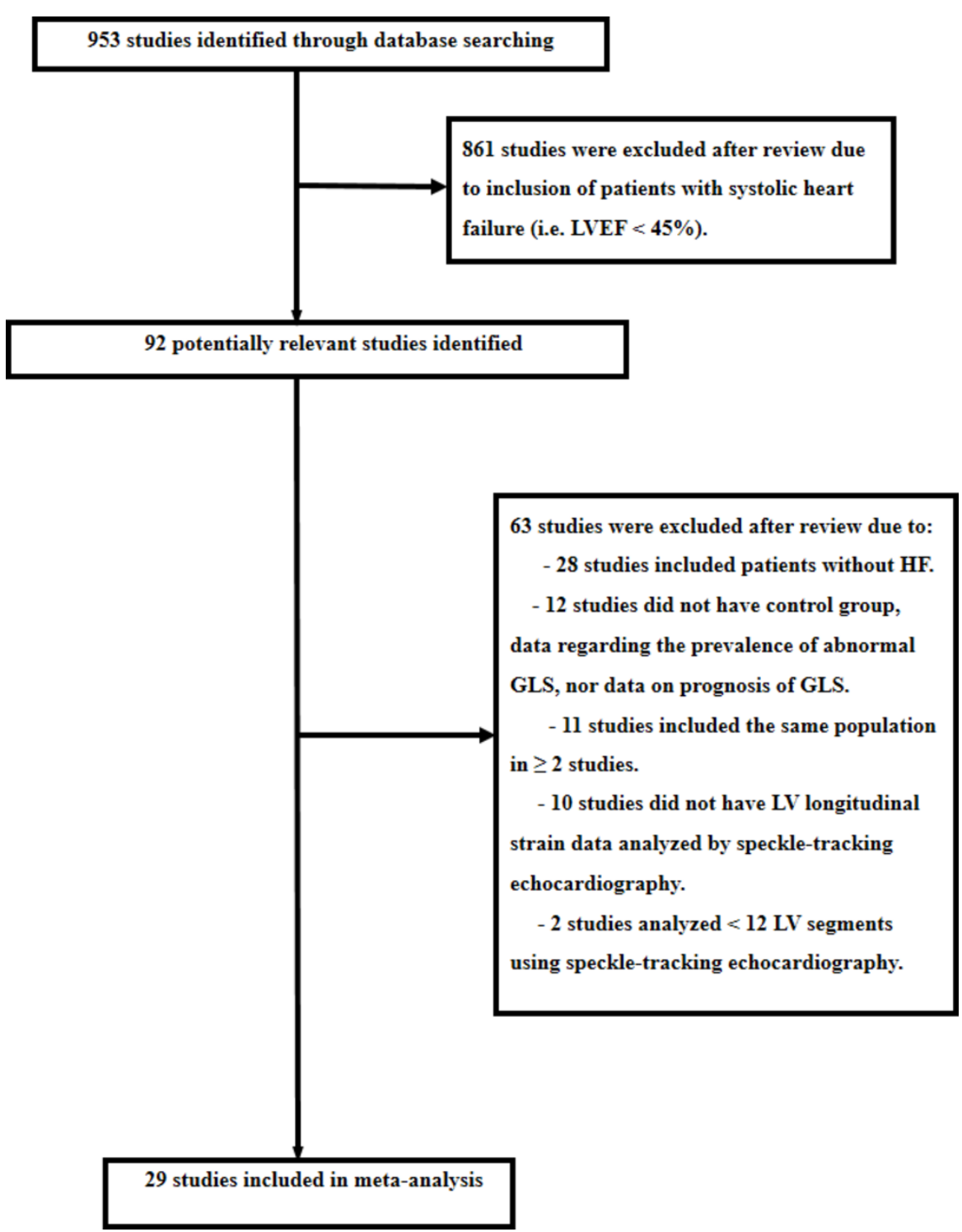

Figure 1 Search process. We searched in different databases (Medline, Embase and Cochrane) published studies until 15 June 2017 that analysed the global longitudinal systolic function of the left ventricular (LV) (global longitudinal systolic strain (GLS)) using two-dimensional speckle-tracking echocardiography in patients with heart failure with preserved ejection fraction. We searched the following Medical Subject Heading terms: 'heart failure', 'echocardiography' and 'strain'. HF, indicates heart failure; LVEF, indicates left ventricular ejection fraction.

Concerning the clinical and LV characteristics of the study population, there were differences between HFpEF and controls regarding comorbidities such as arterial hypertension, diabetes mellitus and history of CAD and regarding LV characteristics such as LV mass and LV filling pressures (table 2). Nonetheless, in a meta-regression analysis, the severity of LV filling pressures was the main factor linked to GLS in patients with HFpEF (see table 3).

\section{LV longitudinal systolic function in HFpEF versus controls}

Patients with HFpEF had significantly lower GLS than control subjects (see table 2 and figures 2 and 3 ). These differences in GLS between HFpEF and controls were significant between patients with $\mathrm{HFpEF}$ and asymptomatic patients (figure 2) as well as between patients with $\mathrm{HFpEF}$ and healthy subjects (figure 3). In line, 19 out of 22 studies showed that patients with HFpEF had significantly lower values of GLS than controls (see figures 2 and 3). On the other hand, there were minimal differences in LVEF between patients with HFpEF and controls and the mean range of LVEF in HFpEF and controls was within the normal range for LVEF (ie, $55 \%-75 \%$ ) (see table 2 and figure 4 ).

In a statistical variability analysis $\left(\mathrm{I}^{2}\right)$, a statistical heterogeneity in GLS values among studies was found (see figures 2 and 3). In this regard, in order to detect the possible sources of statistical heterogeneity on GLS values in the study population, a meta-regression and sensitivity 

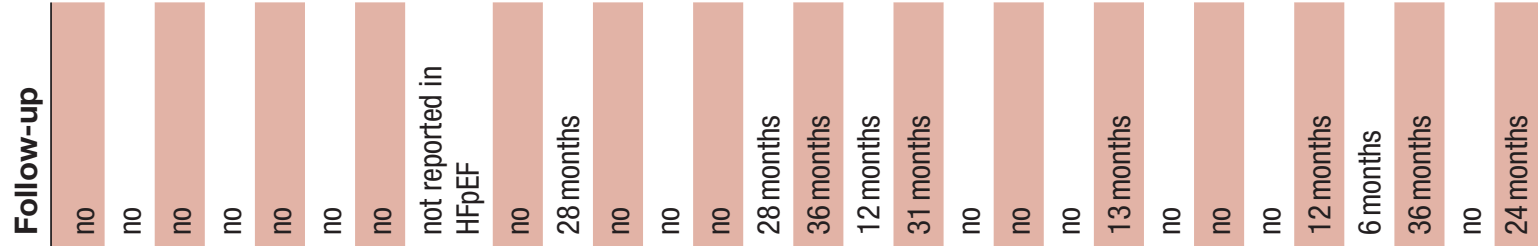

올 을

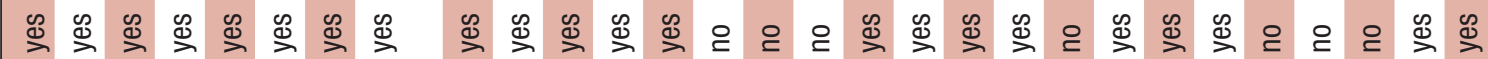

\%

퓨 유

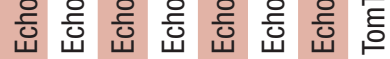

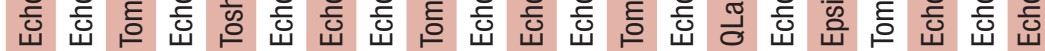

⿹ $\mathrm{d}$ is

s

壱

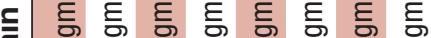

एٓ

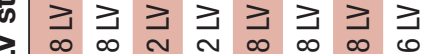

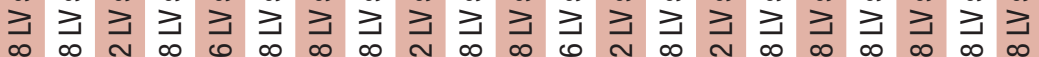

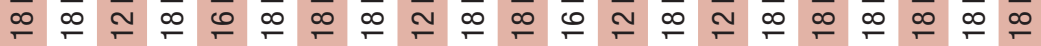

$\frac{\pi}{\frac{\pi}{2}}$

岀 㐫

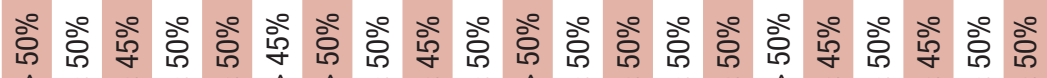

ڤँ $\wedge \wedge \wedge \wedge \wedge \wedge \wedge \wedge \wedge \wedge \wedge \wedge \wedge \wedge \wedge \wedge \wedge \wedge \wedge \wedge \wedge$

का

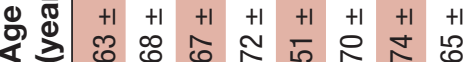

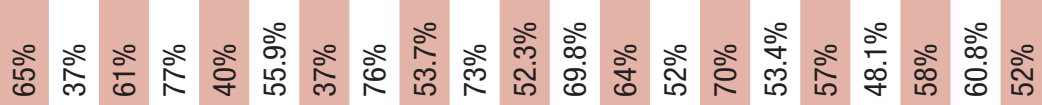

$\frac{\pi}{\frac{\pi}{c}} \frac{\frac{\omega}{\infty}}{\frac{m}{\pi}}$

ํำ

즌.은

造

娄

욷

c)

車

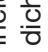

뭉

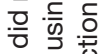

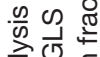

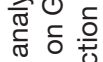

क $\frac{\omega}{0} \cdot \frac{0}{0}$

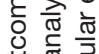

ธิ่

年视

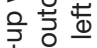

它崫

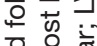

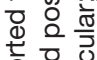

능 은

is

$\stackrel{5}{ \pm}$

둥

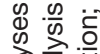

중

我

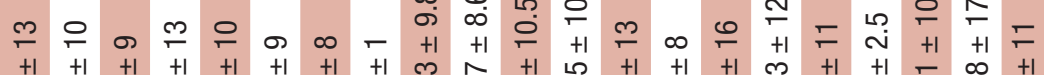

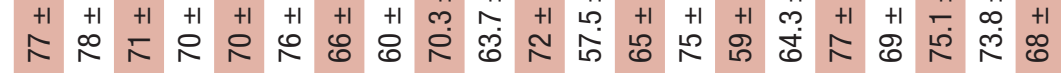

\section{受

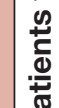 \\ 崖}

क्ष

竞市

ठ

등

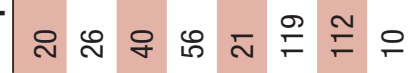

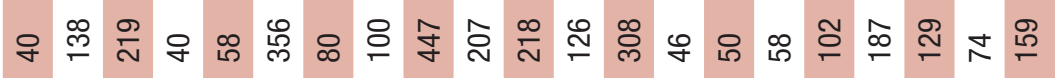

잉 흔 흠

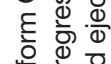

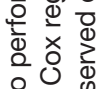

늘

응 을

要爱

¿ ठ

类

논

至

$\stackrel{1}{\mathbb{D}}$

$\stackrel{\vec{\nabla}}{\stackrel{9}{+}}$

$\frac{\sqrt{0}}{\frac{0}{0}}$

क

$\overrightarrow{\vec{\omega}}$

응

$\frac{10}{3}$

$\stackrel{N}{\stackrel{0}{1}}$

용

웅

N

足

$\frac{\mathbb{1}}{3}$

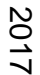

뭉

产

$\frac{0}{10}$

응 剀

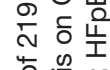


Table 2 Global clinical and echocardiographic characteristics of studies with patients with HFpEF and control subjects

\begin{tabular}{lll}
$\begin{array}{l}\text { Patients with HFpEF } \\
(\mathrm{n}=2284)\end{array}$ & $\begin{array}{l}\text { Asymptomatic patients } \\
(\mathrm{n}=1647)\end{array}$ & $\begin{array}{l}\text { Healthy subjects } \\
(\mathrm{n}=655)\end{array}$ \\
\hline
\end{tabular}

\begin{tabular}{lccl}
\hline Clinical characteristics & & & \\
Age, years & $68.5(51-78)$ & $64.7(47-78)$ & $55(36.5-70)$ \\
\hline Women & $55.2 \%(30 \%-77 \%)$ & $50.9 \%(32.4 \%-77 \%)$ & $58.7 \%(40 \%-70.5 \%)$ \\
\hline Arterial hypertension & $82 \%(40 \%-100 \%)$ & $70.3 \%(8 \%-100 \%)$ & $0 \%$ \\
\hline Diabetes mellitus & $33.4 \%(5 \%-60 \%)$ & $20.8 \%(0 \%-43 \%)$ & $0 \%$ \\
\hline Obesity & $37.8 \%(29.4 \%-58.7 \%)$ & $10.8 \%(8 \%-16.2 \%)$ & $0 \%$ \\
\hline History of CAD & $31.7 \%(0 \%-91.3 \%)$ & $13.6 \%(0 \%-33 \%)$ & $0 \%$ \\
\hline Atrial fibrillation & $8.6 \%(0 \%-73 \%)$ & $0.1 \%(0 \%-1 \%)$ & $0 \%$ \\
\hline Echocardiographic characteristics & & & \\
\hline LV longitudinal systolic strain, $\%$ & $-15.5(-12$ to -18.9$)$ & $-18.3(-15.1$ to - 20.4) & $-19.9(-17.1$ to -21.5) \\
\hline LV ejection fraction, \% & $61.9(58-72)$ & $64(56-71)$ & $63.4(60-67.6)$ \\
\hline LV mass index, g/m² & $105.7(54-144)$ & $85.7(49-115)$ & $78.8(72.7-85)$ \\
\hline LA volume index, $\mathrm{mL} / \mathrm{m}^{2}$ & $37.7(24.8-55)$ & $26.9(16-38)$ & $25.4(18-44)$ \\
\hline Mitral septal-lateral e', cm/s & $5.9(3.4-8)$ & $7.5(4.8-12)$ & $11.1(9-13.5)$ \\
\hline Mitral septal-lateral E/e' ratio & $14.9(10.2-19.9)$ & $10(6.8-12.6)$ & $7.3(6.3-8.5)$ \\
\hline
\end{tabular}

Data are expressed as mean and (range) (ie, the mean value of each variable from all studies as well as the range of the means from all studies). GLS (ie, average longitudinal peak systolic strain from $\geq 12$ LV segments).

CAD, coronary artery disease; GLS, global longitudinal systolic strain; HFpEF, heart failure with preserved ejection fraction;e', septal and lateral annular mitral early diastolic peak velocity using pulsed-TDI; E, mitral inflow early diastolic peak velocity using pulsed Doppler; LA, left atrial.

analysis was performed. In effect, we found that the severity of LV filling pressures (measured by the mitral average septal-lateral $\mathrm{E} / \mathrm{e}$ ' ratio) was the main factor linked to heterogeneity on GLS values among HFpEF studies, whereas the sample size, age and the presence of AF were not significantly linked to GLS (see table 3). In addition, with the purpose of ruling out the possible role of the sample size on GLS values, we performed a

Table 3 Clinical and cardiac factors linked to LV global longitudinal systolic strain (GLS) in patients with HFpEF - Metaregression analysis

\begin{tabular}{lcc} 
& GLS, $\%$ & p Value \\
\cline { 2 - 3 } Clinical and cardiac factors & $\boldsymbol{\beta} \mathbf{( 9 5 \% ~ C l )}$ & 0.32 \\
\hline Age, per 1 year & $-0.05(-0.15$ to 0.05$)$ & $<0.01$ \\
\hline Prevalence of women, per 1\% & $0.08(-0.04$ to 0.12$)$ & 0.41 \\
\hline Prevalence of arterial hypertension, per 1\% & $0.02(-0.03$ to 0.07$)$ & 0.31 \\
\hline Prevalence of diabetes, per 1\% & $-0.02(-0.08$ to 0.02$)$ & $<0.01$ \\
\hline Prevalence of CAD, per 1\% & $-0.04(-0.01$ to -0.07$)$ & 0.27 \\
\hline Prevalence of AF, per 1\% & $-0.02(-0.06$ to 0.01$)$ & 0.03 \\
\hline LVEF, per 1\% & $0.29(0.04$ to 0.53$)$ & 0.05 \\
\hline LV mass, per 1 g/m² & $-0.03(-0.01$ to -0.06$)$ & 0.38 \\
\hline Mitral septal-lateral e', per 1 cm/s & $0.34(-0.40$ to 1.08$)$ & $<0.01$ \\
\hline Mitral septal-lateral E/e', per 1 unit & $-0.39(-0.17$ to -0.61$)$ & 0.53 \\
\hline Sample size of the study, per one patient & $0.01(-0.01$ to 0.02$)$ & \\
\hline
\end{tabular}

The meta-regression analysis was performed in all studies as shown in figures 2 and 3. GLS (ie, average longitudinal peak systolic strain from $\geq 12 \mathrm{LV}$ segments). The $\beta$ coefficient indicates the estimated change in GLS for every estimated change in the independent variable analysed.

AF, atrial fibrillation; CAD, coronary artery disease; GLS, global longitudinal systolic strain; HFpEF, heart failure with preserved ejection fraction; e', septal and lateral annular mitral early diastolic peak velocity using pulsed-TDI; E, mitral inflow early diastolic peak velocity using pulsed Doppler; $\beta$, beta coefficient; LV, left ventricular; LVEF, left ventricular ejection fraction. 
LV Global Longitudinal Systolic Strain (GLS) in Patients with HFpEF vs Asymptomatic Patients

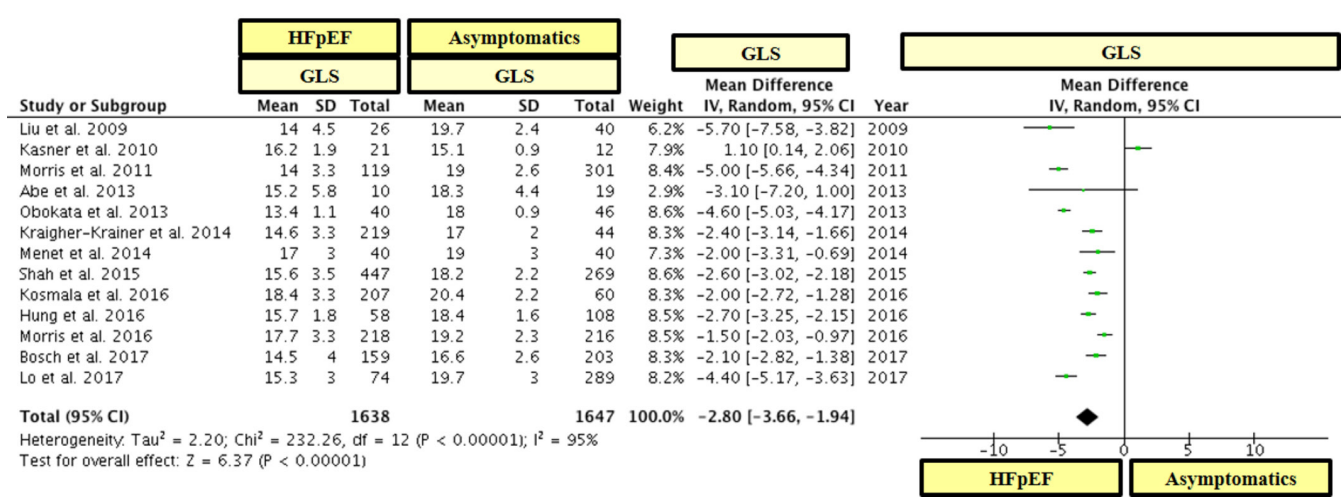

Figure 2 LV global longitudinal systolic strain (GLS) in patients with heart failure with preserved ejection fraction (HFpEF) vs asymptomatic patients. GLS is shown in absolute values.

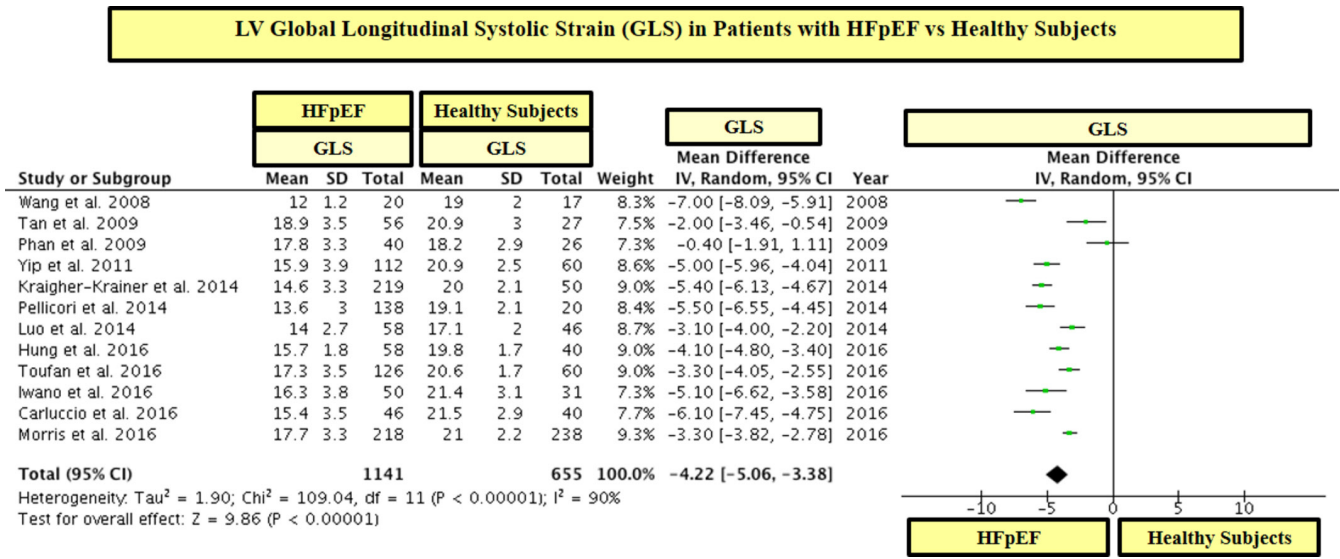

Figure 3 LV global longitudinal systolic strain (GLS) in patients with heart failure with preserved ejection fraction (HFpEF) vs healthy subjects. GLS is shown in absolute values.

subgroup analysis including studies with $\geq 100$ and $<$ 100 patients with HFpEF. In this respect, we found that patients with HFpEF had significantly lower values of GLS than controls in studies that included both $\geq 100$ and $<$ 100 patients with HFpEF (see figures 5 and 6). In addition, in order to exclude the role of AF on the statistical heterogeneity of GLS, we performed a subgroup analysis including only those studies that included patients with HFpEF without AF. In this regard, we found that patients with $\mathrm{HFpEF}$ without AF had also significantly lower values of GLS than controls (see figure 7).

\section{Prevalence of LV Iongitudinal systolic dysfunction in HFpEF}

Regarding the prevalence of LV longitudinal systolic dysfunction in HFpEF, 10 studies (1810 patients with HFpEF and 462 asymptomatic controls) showed that the rate of abnormal GLS was significantly high in patients with HFpEF (mean $65.4 \%$ (range $37 \%-95 \%$ )), whereas in asymptomatic subjects was only of $13 \%$ (range $0 \%-29.6 \%$ ) (table 4). Nonetheless, only one study analysed the clinical and cardiac characteristics of patients with $\mathrm{HFpEF}$ with abnormal GLS. ${ }^{33}$

\section{Prognostic relevance of LV Iongitudinal systolic dysfunction in patients with HFpEF}

Nine studies analysed the prognostic relevance of GLS in patients with HFpEF ( $n=1847$ patients with HFpEF; $n$ of events=620) (see table 5). Four studies showed that GLS was associated with worse CV prognosis, but other five studies did not find any significant association of GLS with outcomes in patients with HFpEF (table 5). Six out of these nine studies analysed the association of GLS with outcomes using only continuous logistic or Cox regression analyses, whereas only three out of these nine studies analysed in a dichotomous analysis the link (ie, OR or HR) of an abnormal GLS to CV outcomes (table 5). Nonetheless, two out of these three studies were multicentre, with large sample size (447 and 348) and high number of events (115 and 177), and showed a significant association of an abnormal GLS with CV outcomes (HR for CV mortality and HF hospitalisation 2.14 (95\% CI 1.26 to 3.66 ) and 1.94 (95\% CI 1.22 to 3.07$)$ ) (see table 5).

\section{Discussion}

In the present study performing a meta-analysis regarding the longitudinal systolic function of the LV analysed by 

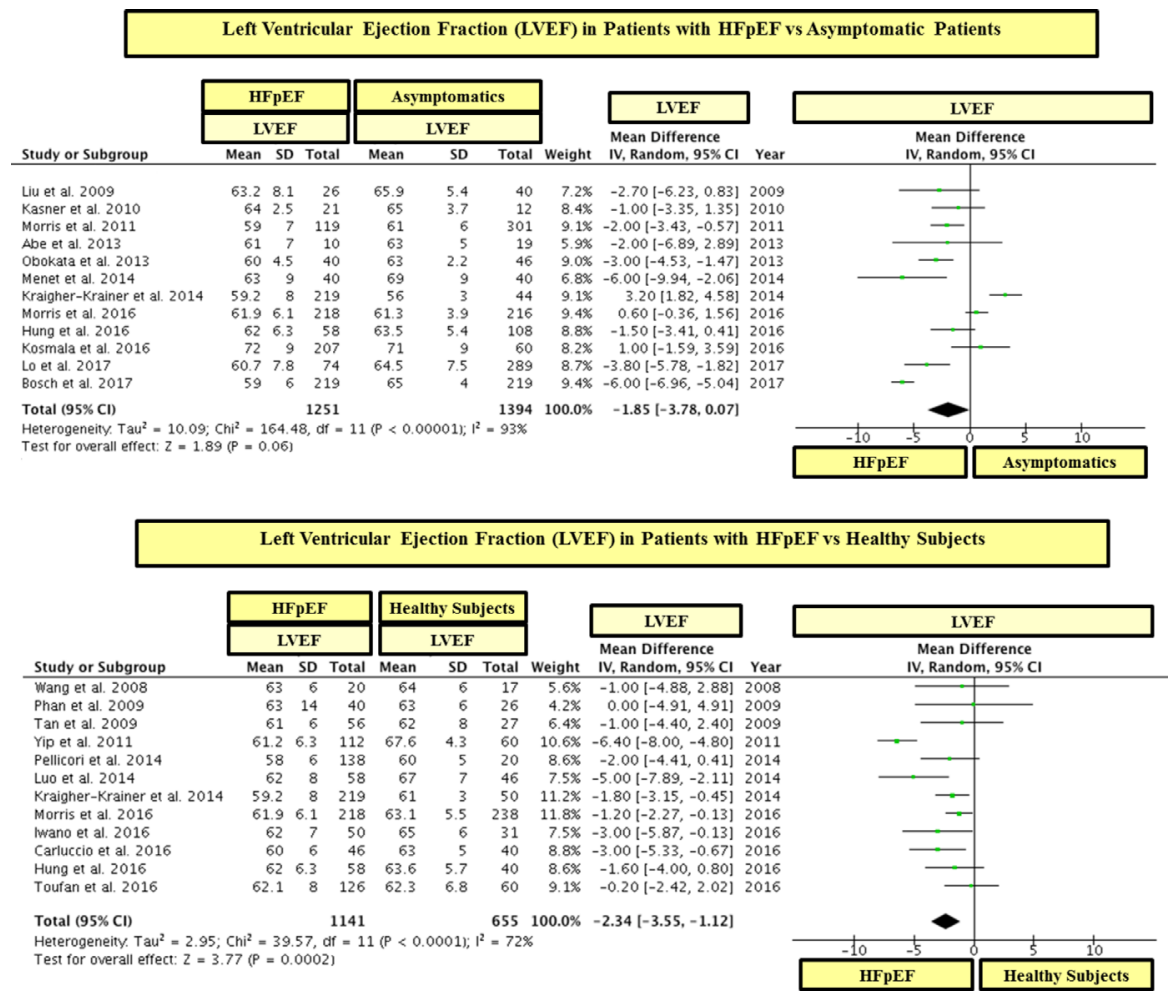

Figure 4 Left ventricular ejection fraction (LVEF) in patients with heart failure with preserved ejection fraction (HFpEF) vs asymptomatic and healthy controls. The study by Shah et $a l^{15}$ was not included in this analysis because the value of LVEF in the control group was not reported.

2DSTE in HFpEF, patients with HFpEF had significantly lower GLS than control subjects and an abnormal GLS was common among patients with HFpEF. Moreover, two large multicentre studies analysing the association of an abnormal GLS with CV outcomes found that an abnormal GLS was significantly linked to CV mortality and HF hospitalisation.

\section{Main findings of this meta-analysis}

On the basis of 22 studies, 2284 patients with HFpEF and 2302 controls, the findings of this meta-analysis confirm that patients with HFpEF have significantly lower LV longitudinal systolic function than asymptomatic controls and that a longitudinal systolic dysfunction of the LV is

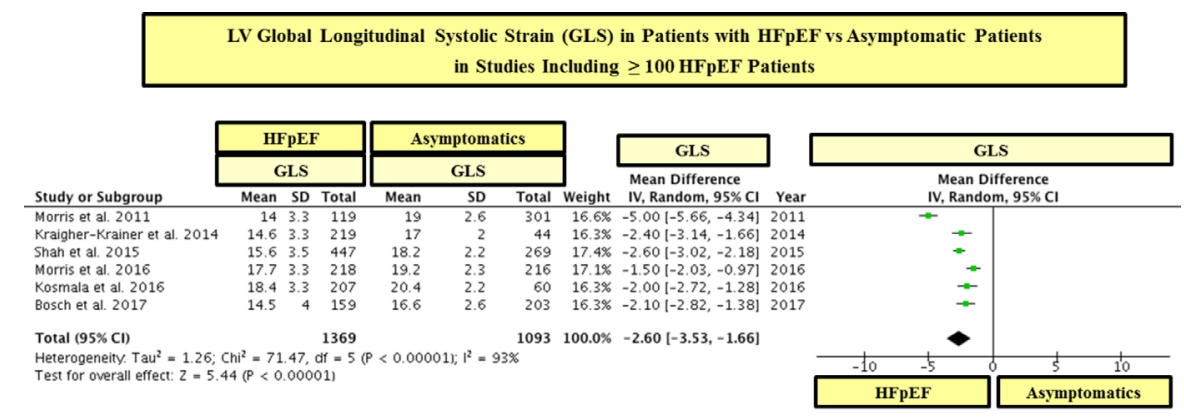

LV Global Longitudinal Systolic Strain (GLS) in Patients with HFpEF vs Healthy Subjects in Studies Including $\geq 100 \mathrm{HFpEF}$ Patients

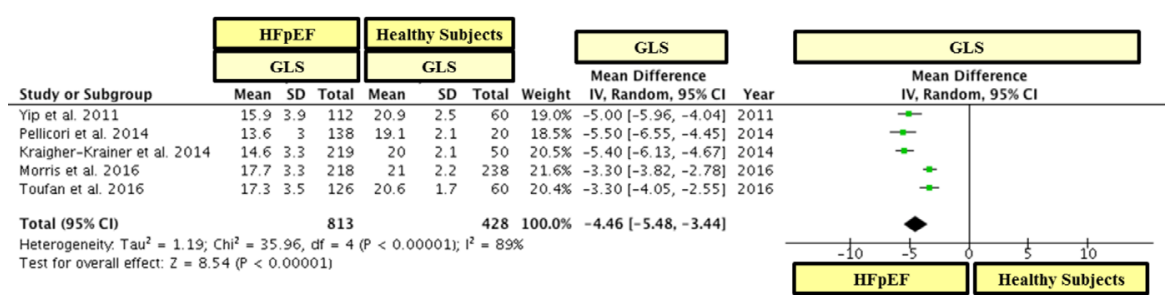

Figure 5 LV global longitudinal systolic strain (GLS) in patients with heart failure with preserved ejection fraction (HFpEF) vs asymptomatic and healthy controls in studies including $\geq 100$ patients with HFpEF. GLS is shown in absolute values. 
LV Global Longitudinal Systolic Strain (GLS) in Patients with HFpEF vs Asymptomatic Patients

in Studies Including < $100 \mathrm{HFpEF}$ Patients

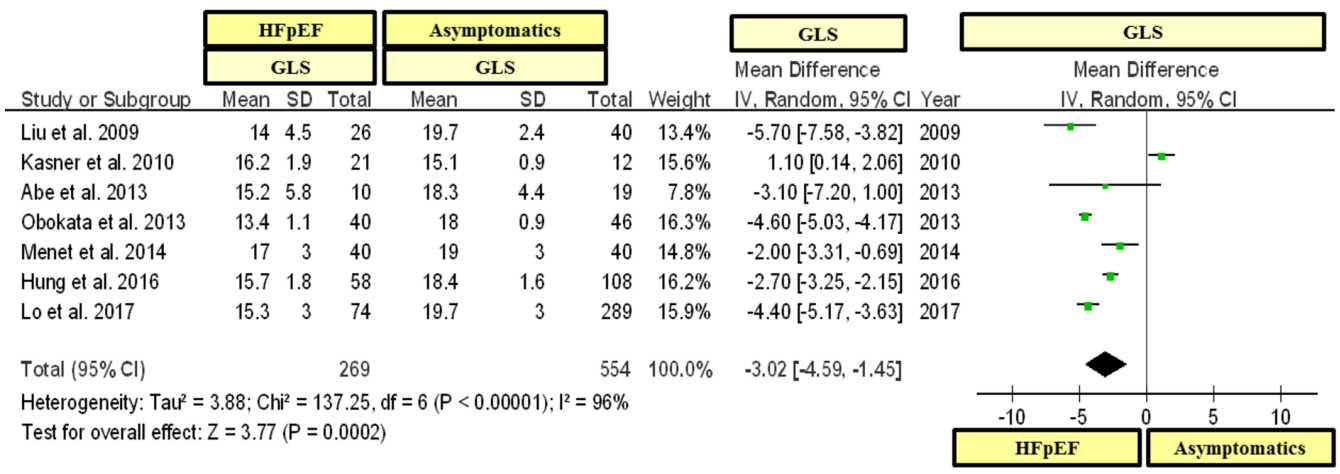

LV Global Longitudinal Systolic Strain (GLS) in Patients with HFpEF vs Healthy Subjects in Studies Including $<100$ HFpEF Patients

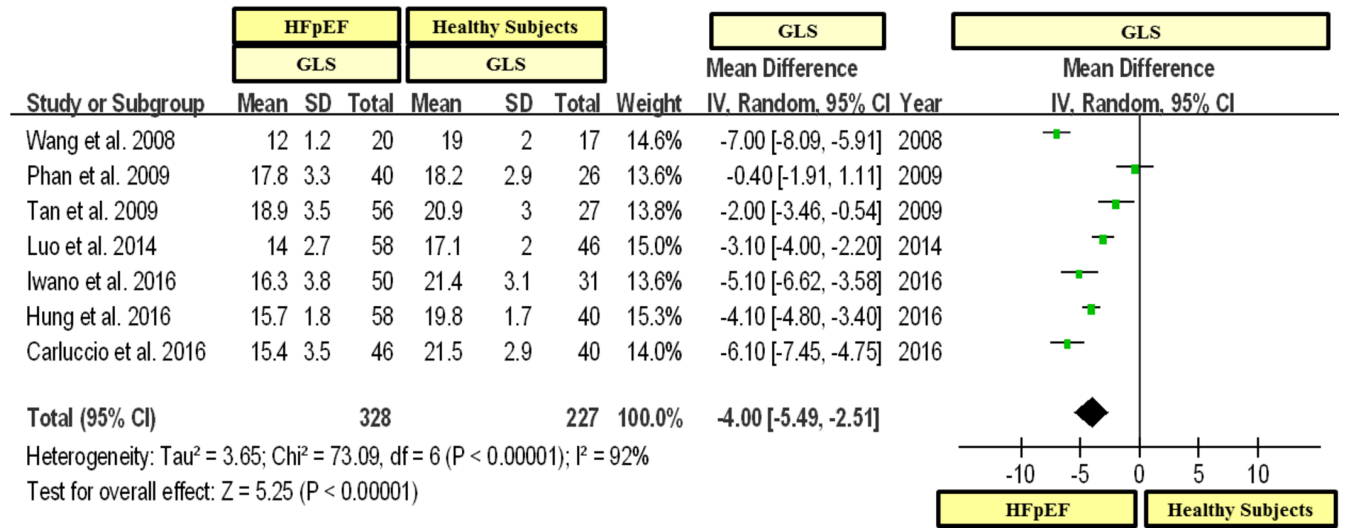

Figure 6 LV global longitudinal systolic strain (GLS) in patients with heart failure with preserved ejection fraction (HFpEF) vs asymptomatic and healthy controls in studies including $<100$ patients with HFpEF. GLS is shown in absolute values.

common among patients with HFpEF. Nonetheless, despite the fact that the number of studies and patients was large, the amount of studies reporting the characteristics of patients with abnormal GLS as well as the prognostic consequences of an abnormal GLS was lower. In fact, only one study analysed the clinical and cardiac characteristics of patients with HFpEF with abnormal GLS and only two large multicentre studies analysed in a dichotomous analysis the association of an abnormal GLS with CV outcomes. ${ }^{152} 23$ Accordingly, on the basis of this meta-analysis, we can confirm that the longitudinal systolic function of the $\mathrm{LV}$ is altered in high proportion of patients with HFpEF, but the clinical and cardiac characteristics of this subgroup of patients as well as the clinical consequences of LV longitudinal systolic dysfunction in patients with HFpEF need to be confirmed.

While nine studies have analysed the association of the longitudinal systolic function of the LV (analysed by GLS) with CV outcomes in patients with HFpEF, ${ }^{14} 152024$ 29-33 only two of these studies were multicentric, enrolled large number of patients (>300) and had high number of events $(>100) .{ }^{15}{ }^{20}$ In this regard, Shah et $a l^{15}$ analysing the echocardiographic data of the TOPCAT trial found that an abnormal GLS was significantly linked to worse $\mathrm{CV}$ outcomes (CV death and HF hospitalisation) in patients with HFpEF. In agreement, Donal et $a l^{20}$ analysing the echocardiographic data of the KaRen study found a significant association of an abnormal GLS with CV outcomes. However, other two smaller multicentre studies and three singlecentre studies did not find any significant association of GLS with outcomes in HFpEF. ${ }^{29-33}$ Nonetheless, it is important to highlight that the analyses in the TOPCAT and KaRen studies were dichotomous analyses (ie, analysing the HR of an abnormal GLS with CV outcomes), ${ }^{1520}$ whereas the other smaller studies analysed the association of GLS with CV outcomes using only continuous logistic or Cox regression analyses. ${ }^{29-33}$ Accordingly, while it is not possible to confirm in this meta-analysis if an abnormal GLS is linked to worse CV outcomes in HFpEF, we consider that further large multicentre studies with the aim to confirm the prognostic role of abnormal GLS in HFpEF are warranted. 
LV Global Longitudinal Systolic Strain (GLS) in Patients with HFpEF without Atrial Fibrillation vs Asymptomatic Patients

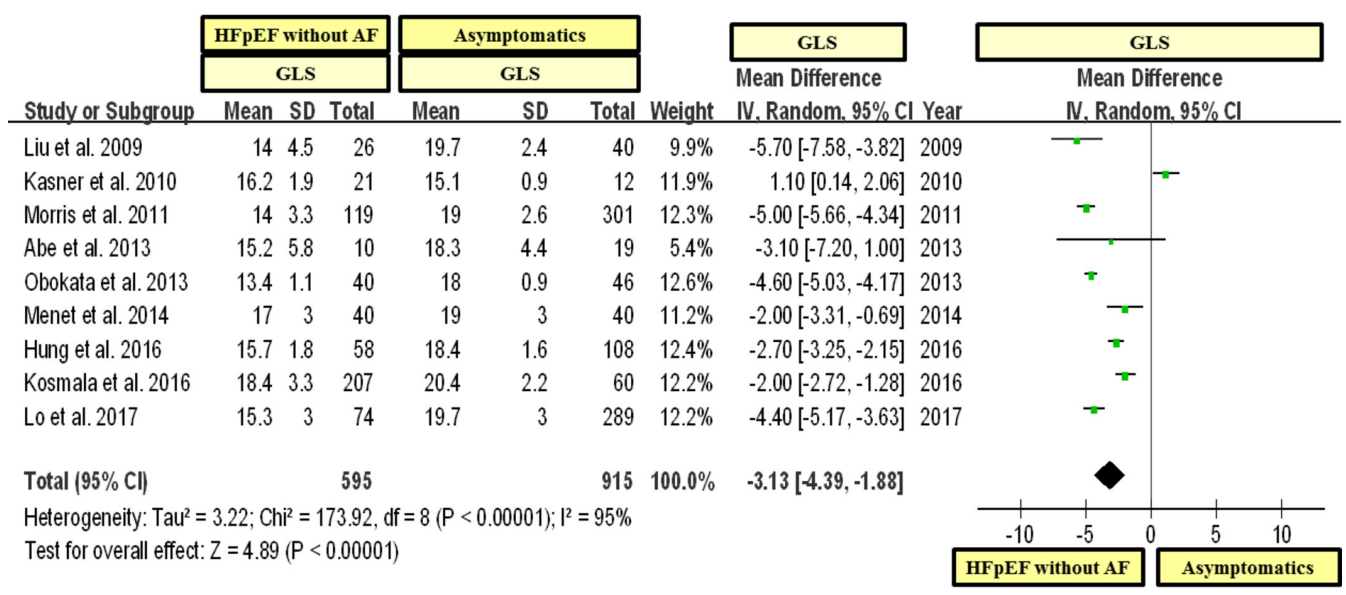

LV Global Longitudinal Systolic Strain (GLS) in Patients with HFpEF without Atrial Fibrillation vs Healthy Subjects

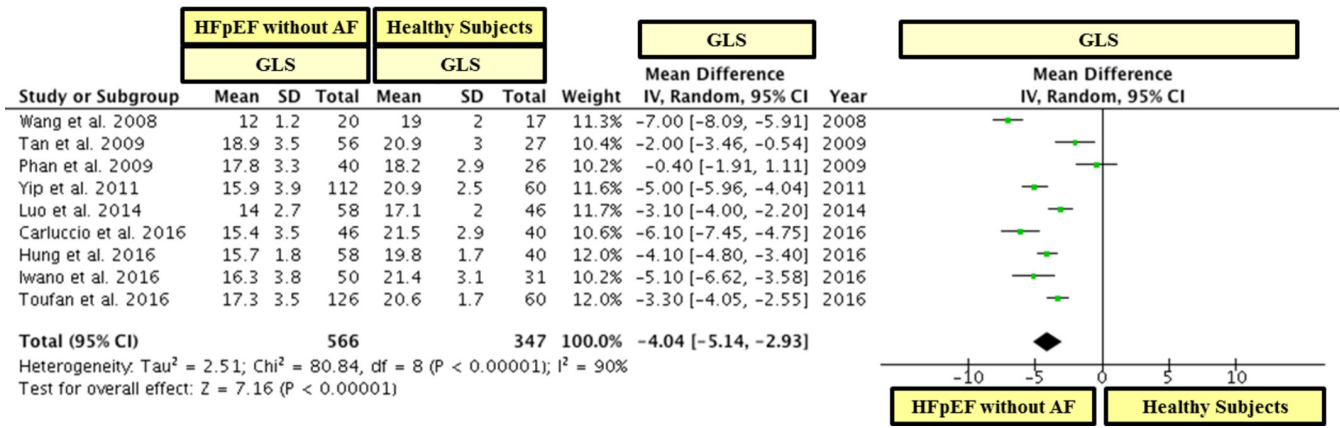

Figure 7 LV global longitudinal systolic strain (GLS) in patients with heart failure with preserved ejection fraction (HFpEF) without atrial fibrillation vs asymptomatic and healthy controls. GLS is shown in absolute values.

Table 4 Prevalence of LV longitudinal systolic dysfunction in patients with HFpEF vs controls

\begin{tabular}{|c|c|c|c|c|c|}
\hline Study & $\begin{array}{l}\text { HFpEF patients rate of } \\
\text { abnormal GLS }\end{array}$ & $\begin{array}{l}\text { Asymptomatic } \\
\text { controls rate of } \\
\text { abnormal GLS }\end{array}$ & $\begin{array}{l}\text { Cut-off of } \\
\text { abnormal GLS }\end{array}$ & $\begin{array}{l}\text { LV segments } \\
\text { analysed }\end{array}$ & $\begin{array}{l}\text { Software } \\
\text { package }\end{array}$ \\
\hline Wang et $a l^{4}$ & $95 \%$ & $5 \%$ & $-16 \%$ & 18 & EchoPac \\
\hline Liu et $a l^{5}$ & $85 \%$ & $15 \%$ & $-17.5 \%$ & 18 & EchoPac \\
\hline Morris et $a l^{7}$ & $81.5 \%$ & $15.5 \%$ & $-16 \%$ & 18 & EchoPac \\
\hline Yip et $a l^{8}$ & $37 \%$ & $0 \%$ & $-16 \%$ & 18 & EchoPac \\
\hline Donal et $a l^{19}$ & $39 \%$ & No control group & $-16 \%$ & 18 & EchoPac \\
\hline Shah et $a l^{15}$ & $52 \%$ & Not reported & $-15.8 \%$ & 12 & TomTec \\
\hline Freed et $a l^{31}$ & $75 \%$ & No control group & $-20 \%$ & 12 & TomTec \\
\hline DeVore et $a l^{33}$ & $65 \%$ & No control group & $-16 \%$ & 18 & TomTec \\
\hline
\end{tabular}

The rate of abnormal GLS indicates the prevalence of LV longitudinal systolic dysfunction. GLS (ie, average longitudinal peak systolic strain from $\geq 12$ LV segments).

HFpEF, heart failure with preserved ejection fraction; GLS, global longitudinal systolic strain. 


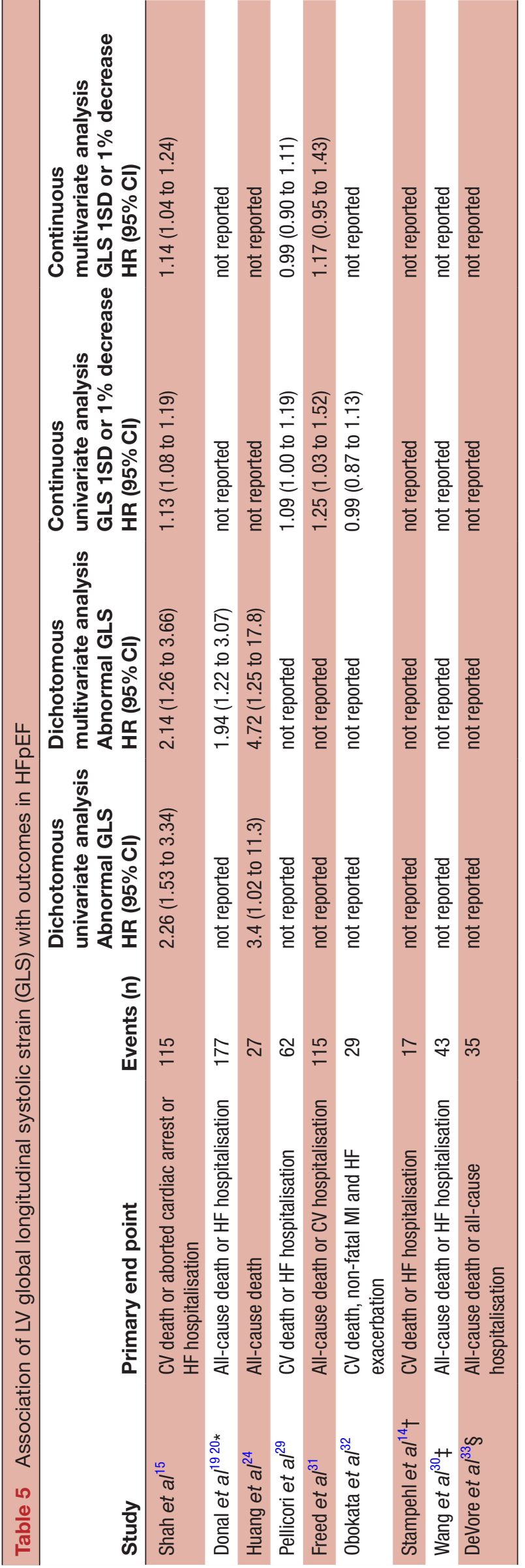

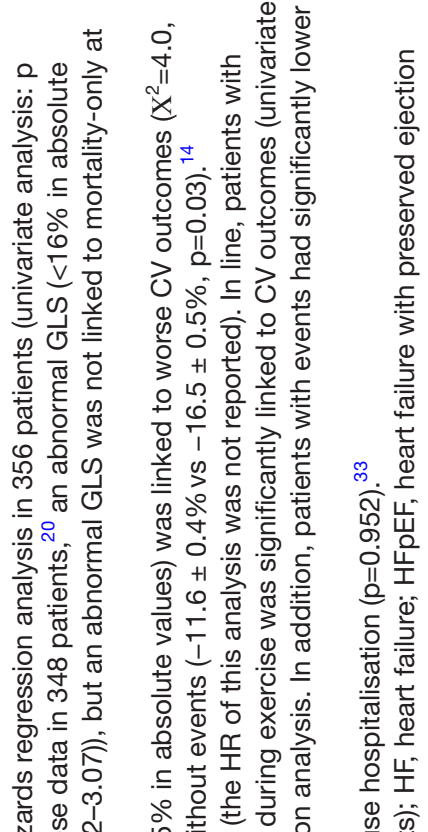

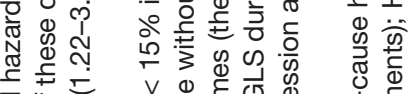

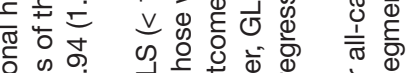

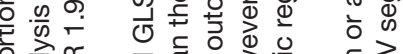

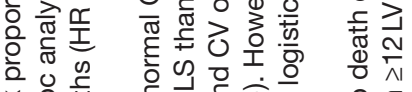

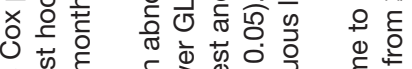

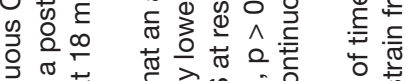

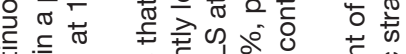

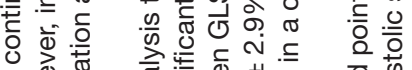

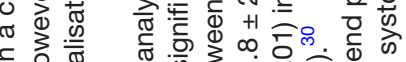

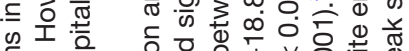

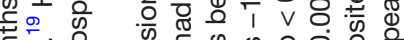

후웡

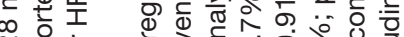

N

क人 d

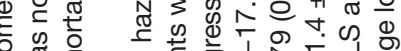

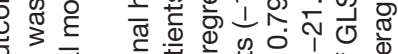

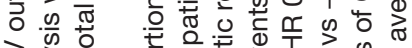

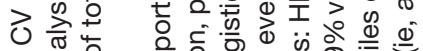

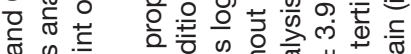

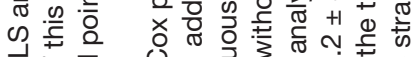

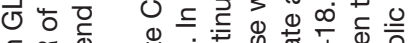

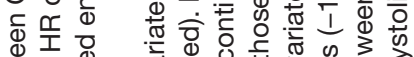

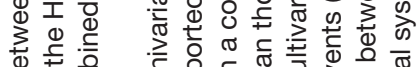

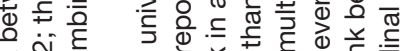

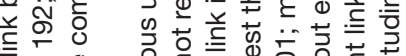

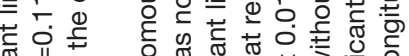

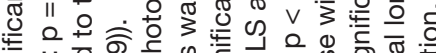

旁

क

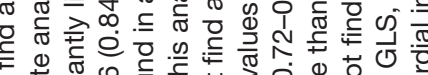

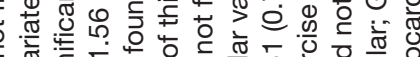

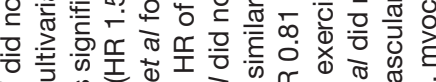

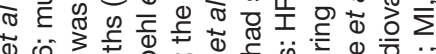

बै

* 


\section{Clinical perspectives on the basis of the findings of this meta- analysis}

Isolated LV diastolic dysfunction (ie, abnormalities of LV myocardial stiffness and relaxation with normal LVEF) has long been considered the main underlying mechanism in HFpEF. ${ }^{1-3}$ On the basis of this pathophysiological model, several clinical trials have been conducted to restore the diastolic function of the $\mathrm{LV}$ in patients with HFpEF in order to improve the prognosis of these patients. ${ }^{35} 36$ However, none of these treatments has been shown to decrease mortality in patients with HFpEF. ${ }^{35} 36$ For this reason, additional pathophysiological mechanisms should be taken into consideration in the design of new clinical trials in this heterogeneous disease. The present meta-analysis analysing 2284 patients with $\mathrm{HFpEF}$ and 2302 controls confirms that the longitudinal systolic function of the LV is significantly altered in high proportion of patients with HFpEF. In addition, two large multicentre studies showed that an abnormal LV longitudinal systolic function is significantly linked to CV mortality and HF hospitalisation in these patients. ${ }^{15} 20$ Therefore, we consider that further large multicentre studies with the aim to validate the prognostic relevance of an abnormal GLS in patients with HFpEF are warranted, because if the prognostic role of this LV systolic alteration is confirmed, a future therapeutic target could arise on this complex disease, for which, so far, no effective therapies exist.

\section{LIMITATIONS}

Some considerations should be taken into account on this meta-analysis. Given that GLS values could vary among different software packages, ${ }^{37}$ we consider that the cut-off of GLS used to define LV longitudinal systolic dysfunction should be considered according to the ultrasound software package used in each study. In addition, it is worth to note that GLS, like other 2D methods such as LVEF, depends on the imaging quality and for these reasons the patients included in all studies of this meta-analysis had adequate imaging quality for an analysis by 2DSTE. Hence, the results of this meta-analysis could not be extrapolated to patients with poor imaging quality of the LV. Furthermore, while in the present meta-analysis were analysed all published studies that analysed GLS in HFpEF, there was some statistical heterogeneity in GLS values in the study population. In this respect, we performed a meta-regression analysis in order to detect the possible sources of statistical heterogeneity on GLS values among the studies. In effect, we found that the severity of LV filling pressures was the main factor linked to heterogeneity on GLS values among HFpEF studies, whereas the sample size, age and the presence of AF were not linked to GLS values. Nonetheless, it is important to note that it was not possible to perform a subgroup analysis including studies with HFpEF without history of CAD because only one study excluded patients with history of CAD. ${ }^{21}$

\section{CONCLUSIONS}

The present meta-analysis analysing 2284 patients with HFpEF and 2302 controls confirms that the longitudinal systolic function of the LV is significantly altered in high proportion of patients with HFpEF. In addition, two large multicentre studies showed that an abnormal LV longitudinal systolic function is significantly linked to CV mortality and HF hospitalisation in these patients. Therefore, we consider that further large multicentre studies with the aim to validate the prognostic relevance of an abnormal GLS in patients with HFpEF are warranted, because if the prognostic role of this LV systolic alteration is confirmed, a future therapeutic target could arise on this complex disease, for which, so far, no effective therapies exist.

\section{Author affiliations}

${ }^{1}$ Department of Internal Medicine and Cardiology, Charité - Universitätsmedizin Berlin, DZHK (German Centre for Cardiovascular Research) partner site Berlin and Berlin Institute of Health (BIH), Berlin, Germany

${ }^{2}$ Department of Ultrasound in Medicine, Shanghai Jiao Tong University Affiliated Sixth People's Hospital, Shanghai Institute of Ultrasound in Medicine, Shanghai, China

${ }^{3}$ Department of Internal Medicine and Cardiology, Meoclinic, Berlin, Germany ${ }^{4}$ Department of Radiology, Private Clinic of Radiology (Q-Diagnostica-Scanner Murcia), Murcia, Spain

${ }^{5}$ Department of Cardiology, CHU Rennes, Pontchaillou Hospital, Rennes, France ${ }^{6}$ Department of Internal Medicine and Cardiology, German Heart Institute, Berlin, Germany

Contributors All authors have contributed significantly to this study and meet the criteria for authorship.

Competing interests None declared.

Patient consent Obtained in all studies.

Ethics approval Charite University Hospital.

Provenance and peer review Not commissioned; externally peer reviewed.

Data sharing statement There are no additional data available for this paper.

Open Access This is an Open Access article distributed in accordance with the Creative Commons Attribution Non Commercial (CC BY-NC 4.0) license, which permits others to distribute, remix, adapt, build upon this work non-commercially, and license their derivative works on different terms, provided the original work is properly cited and the use is non-commercial. See: http://creativecommons.org/ licenses/by-nc/4.0/

(C) Article author(s) (or their employer(s) unless otherwise stated in the text of the article) 2017. All rights reserved. No commercial use is permitted unless otherwise expressly granted.

\section{REFERENCES}

1. Zile MR, Baicu CF, Gaasch WH. Diastolic heart failure-abnormalities in active relaxation and passive stiffness of the left ventricle. $N$ Engl J Med 2004;350:1953-9.

2. Brucks S, Little WC, Chao T, et al. Contribution of left ventricular diastolic dysfunction to heart failure regardless of ejection fraction. Am J Cardiol 2005;95:603-6.

3. Westermann D, Kasner M, Steendijk P, et al. Role of left ventricular stiffness in heart failure with normal ejection fraction. Circulation 2008;117:2051-60.

4. Wang J, Khoury DS, Yue Y, et al. Preserved left ventricular twist and circumferential deformation, but depressed longitudinal and radial deformation in patients with diastolic heart failure. Eur Heart $J$ 2008;29:1283-9.

5. Liu YW, Tsai WC, Su CT, et al. Evidence of left ventricular systolic dysfunction detected by automated function imaging in patients with heart failure and preserved left ventricular ejection fraction. J Card Fail 2009;15:782-9. 
6. Tan YT, Wenzelburger F, Lee E, et al. The pathophysiology of heart failure with normal ejection fraction: exercise echocardiography reveals complex abnormalities of both systolic and diastolic ventricular function involving torsion, untwist, and longitudinal motion. J Am Coll Cardiol 2009;54:36-46.

7. Morris DA, Gailani M, Vaz Pérez A, et al. Left atrial systolic and diastolic dysfunction in heart failure with normal left ventricular ejection fraction. J Am Soc Echocardiogr 2011;24:651-62.

8. Yip GW, Zhang Q, Xie JM, et al. Resting global and regional left ventricular contractility in patients with heart failure and normal ejection fraction: insights from speckle-tracking echocardiography. Heart 2011;97:287-94.

9. Abe H, Caracciolo G, Kheradvar A, et al. Contrast echocardiography for assessing left ventricular vortex strength in heart failure: a prospective cohort study. Eur Heart J Cardiovasc Imaging 2013;14:1049-60.

10. Obokata M, Negishi K, Kurosawa K, et al. Incremental diagnostic value of la strain with leg lifts in heart failure with preserved ejection fraction. JACC Cardiovasc Imaging 2013;6:749-58.

11. Kraigher-Krainer E, Shah AM, Gupta DK, et al. Impaired systolic function by strain imaging in heart failure with preserved ejection fraction. J Am Coll Cardiol 2014;63:447-56.

12. Menet A, Greffe L, Ennezat PV, et al. Is mechanical dyssynchrony a therapeutic target in heart failure with preserved ejection fraction? Am Heart J 2014;168:909-16.

13. Luo XX, Fang F, Lee AP, et al. What can three-dimensional speckletracking echocardiography contribute to evaluate global left ventricular systolic performance in patients with heart failure? Int $J$ Cardiol 2014;172:132-7.

14. Stampehl MR, Mann DL, Nguyen JS, et al. Speckle strain echocardiography predicts outcome in patients with heart failure with both depressed and preserved left ventricular ejection fraction. Echocardiography 2015;32:71-8

15. Shah AM, Claggett B, Sweitzer NK, et al. Prognostic Importance of Impaired Systolic Function in Heart Failure With Preserved Ejection Fraction and the Impact of Spironolactone. Circulation 2015;132:402-14

16. Kosmala W, Rojek A, Przewlocka-Kosmala M, et al. Contributions of nondiastolic factors to exercise intolerance in heart failure with preserved ejection fraction. J Am Coll Cardiol 2016;67:659-70.

17. Morris DA, Krisper M, Nakatani S, et al. Normal range and usefulness of right ventricular systolic strain to detect subtle right ventricular systolic abnormalities in patients with heart failure: a multicentre study. Eur Heart J Cardiovasc Imaging 2017;18:212-23.

18. Toufan M, Mohammadzadeh Gharebaghi S, Pourafkari L, et al. Systolic longitudinal function of the left ventricle assessed by speckle tracking in heart failure patients with preserved ejection fraction. J Tehran Heart Cent 2015;10:194-200.

19. Donal E, Lund LH, Oger E, et al. New echocardiographic predictors of clinical outcome in patients presenting with heart failure and a preserved left ventricular ejection fraction: a subanalysis of the Ka (Karolinska) Ren (Rennes) Study. Eur J Heart Fail 2015;17:680-8.

20. Donal E, Lund LH, Oger E, et al. Importance of combined left atrial size and estimated pulmonary pressure for clinical outcome in patients presenting with heart failure with preserved ejection fraction. Eur Heart J Cardiovasc Imaging 2017;18:629-35.

21. Carluccio E, Biagioli P, Zuchi C, et al. Fibrosis assessment by integrated backscatter and its relationship with longitudinal deformation and diastolic function in heart failure with preserved ejection fraction. Int J Cardiovasc Imaging 2016;32:1071-80.
22. Iwano H, Kamimura D, Fox ER, et al. Presence and implication of temporal nonuniformity of early diastolic left ventricular wall expansion in patients with heart failure. J Card Fail 2016;22:945-53.

23. Hung $\mathrm{CL}$, Yun $\mathrm{CH}$, Lai $\mathrm{YH}$, et al. An observational study of the association among interatrial adiposity by computed tomography measure, insulin resistance, and left atrial electromechanical disturbances in heart failure. Medicine 2016;95:e3912.

24. Huang W, Chai SC, Lee SGS, et al. Prognostic Factors After Index Hospitalization for Heart Failure With Preserved Ejection Fraction. Am J Cardiol 2017;119:2017-20.

25. Ci L, Lai YH, Chang SN, et al. The associations among co-morbidity, cardiac geometries and mechanics in hospitalized heart failure with or without preserved ejection fraction. Clin Exp Hypertens 2017;1:1-8.

26. Bosch L, Lam CSP, Gong L, et al. Right ventricular dysfunction in left-sided heart failure with preserved versus reduced ejection fraction. Eur J Heart Fail 2017.

27. Phan TT, Shivu GN, Abozguia K, et al. Left ventricular torsion and strain patterns in heart failure with normal ejection fraction are similar to age-related changes. Eur J Echocardiogr 2009;10:793-800.

28. Kasner M, Gaub R, Sinning D, et al. Global strain rate imaging for the estimation of diastolic function in HFNEF compared with pressurevolume loop analysis. Eur J Echocardiogr 2010;11:743-51.

29. Pellicori P, Kallvikbacka-Bennett A, Khaleva O, et al. Global longitudinal strain in patients with suspected heart failure and a normal ejection fraction: does it improve diagnosis and risk stratification? Int J Cardiovasc Imaging 2014;30:69-79.

30. Wang J, Fang F, Wai-Kwok Yip G, et al. Left ventricular long-axis performance during exercise is an important prognosticator in patients with heart failure and preserved ejection fraction. Int $J$ Cardiol 2015;178:131-5

31. Freed BH, Daruwalla V, Cheng JY, et al. Prognostic utility and clinical significance of cardiac Mechanics in heart failure with preserved ejection fraction: importance of left atrial strain. Circ Cardiovasc Imaging 2016;9:e003754.

32. Obokata M, Takeuchi M, Negishi K, et al. Relation Between Echocardiogram-Based Cardiac Parameters and Outcome in Heart Failure With Preserved and Reduced Ejection Fraction. Am J Cardiol 2016;118:1356-62.

33. DeVore AD, McNulty S, Alenezi F, et al. Impaired left ventricular global longitudinal strain in patients with heart failure with preserved ejection fraction: insights from the RELAX trial. Eur $J$ Heart Fail 2017; 19:893-900.

34. Stewart LA, Clarke M, Rovers $M$, et al. Preferred Reporting Items for Systematic Review and Meta-Analyses of individual participant data: the PRISMA-IPD Statement. JAMA 2015;313:1657-65.

35. Zhang $Q$, Chen $Y$, Liu $Q$, et al. Effects of renin-angiotensinaldosterone system inhibitors on mortality, hospitalization, and diastolic function in patients with HFpEF. A meta-analysis of 13 randomized controlled trials. Herz 2016;41:76-86.

36. Bavishi $\mathrm{C}$, Chatterjee $\mathrm{S}$, Ather $\mathrm{S}$, et al. Beta-blockers in heart failure with preserved ejection fraction: a meta-analysis. Heart Fail Rev 2015;20:193-201.

37. Takigiku K, Takeuchi M, Izumi C, et al. Normal range of left ventricular 2-dimensional strain: Japanese Ultrasound Speckle Tracking of the Left Ventricle (JUSTICE) study. Circ J 2012;76:2623-32.

38. Biaggi P, Carasso S, Garceau P, et al. Comparison of two different speckle tracking software systems: does the method matter? Echocardiography 2011;28:539-47. 